\title{
Plasma folate and its association with folic acid supplementation, socio-demographic and lifestyle factors among New Zealand pregnant women
}

\author{
Rhodi E. Bulloch ${ }^{1 *}$, Lesley M. E. McCowan² ${ }^{2}$ John M. D. Thompson ${ }^{3}$, Lisa A. Houghton ${ }^{4}$ and \\ Clare R. Wall ${ }^{1}$ \\ ${ }^{1}$ Discipline of Nutrition and Dietetics, The University of Auckland, Auckland 1142, New Zealand \\ ${ }^{2}$ Department of Obstetrics and Gynaecology, The University of Auckland, Auckland 1142, New Zealand \\ ${ }^{3}$ Department of Paediatrics, Child and Youth Health, The University of Auckland, Auckland 1142, New Zealand \\ ${ }^{4}$ Department of Human Nutrition, University of Otago, Dunedin 9054, New Zealand
}

(Submitted 21 January 2019 - Final revision received 30 June 2019 - Accepted 12 July 2019)

\section{Abstract}

Folic acid (FA) supplementation is recommended in the periconceptional period, for the prevention of neural tube defects. Limited data are available on the folate status of New Zealand (NZ) pregnant women and its association with FA supplementation intake. Objectives were to examine the relationship between plasma folate (PF) and reported FA supplement use at 15 weeks' gestation and to explore socio-demographic and lifestyle factors associated with PF. We used data and blood samples from NZ participants of the Screening for Pregnancy Endpoints cohort study. Healthy nulliparous women with singleton pregnancy ( $n$ 1921) were interviewed and blood samples collected. PF was analysed via microbiological assay. Of the participants, $73 \%$ reported taking an FA supplement at 15 weeks' gestation - of these, $79 \%$ were taking FA as part of/alongside a multivitamin supplement. Of FA supplement users, $56 \%$ reported consuming a daily dose of $\geq 800 \mu \mathrm{g}$; $39 \%$ reported taking less than $400 \mu \mathrm{g} / \mathrm{d}$. Mean PF was significantly higher in women reporting FA supplementation (54.6 (se 1.5) nmol/1) $v$. no FA supplementation $(35.1$ (SE 1.6$) \mathrm{nmol} / \mathrm{l})(P<0 \cdot 0001)$. Reported daily FA supplement dose and PF were significantly positively correlated $(r 0 \cdot 41$; $P<0.05)$. Younger maternal age, Pacific and Maori ethnicity and obesity were negatively associated with PF levels; vegetarianism was positively associated with PF. Reported FA supplement dose was significantly associated with PF after adjustment for socio-demographic, lifestyle confounders and multivitamin intake. The relationship observed between FA supplementation and PF demonstrates that self-reported intake is a reliable proxy for FA supplement use in this study population.

\section{Key words: Folic acid: Folate: Supplementation: Pregnancy: Plasma folate: Biomarkers}

Folate is an essential nutrient in pregnancy due to its role as a methyl group donor in one-carbon metabolism and its involvement in DNA synthesis, replication and methylation ${ }^{(1-5)}$. It is also important for the re-methylation of homocysteine, converting it to methionine, helping to control plasma homocysteine levels ${ }^{(1,4,6)}$. Folate requirements are increased during pregnancy $^{(7)}$ and an adequate supply is essential for the healthy development of the placenta and fetus ${ }^{(3,5,8)}$.

Inadequate intake of folate in pregnancy is associated with poor folate status and elevated levels of homocysteine ${ }^{(9)}$. Epidemiological evidence has shown that maternal folic acid (FA) supplementation during pregnancy helps to protect against poor folate status and elevated homocysteine levels, which are associated with adverse outcomes such as pre-eclampsia ${ }^{(2,5,10)}$, spontaneous preterm birth ${ }^{(11-14)}$ and small-for-gestational age babies $^{(5,15-20)}$. These adverse outcomes are an important cause of maternal and fetal morbidity and mortality worldwide ${ }^{(11,21-23)}$.

The WHO recommends maternal FA supplementation (400 $\mu \mathrm{g} / \mathrm{d}$ ) in the pre-conception period and for the first 12 weeks of pregnancy for the prevention of neural tube defects (NTD), and that this should continue for the duration of pregnancy to ensure best pregnancy outcomes ${ }^{(24)}$. In comparison, the New Zealand (NZ) Ministry of Health ${ }^{(25)}$ recommends that women planning pregnancy should take $800 \mu \mathrm{g}$ FA daily for at least 4 weeks prior to conception and continue taking FA for the first 12 weeks of gestation ${ }^{(25)}$. FA supplementation $(800 \mu \mathrm{g} / \mathrm{d})$ is

Abbreviations: FA, folic acid; NTD, neural tube defect; NZ, New Zealand; PF, plasma folate; QC, quality control; SCOPE, Screening for Pregnancy Endpoints; SEI, socio-economic index.

* Corresponding author: R. E. Bulloch, email r.bulloch@auckland.ac.nz 
currently subsidised in NZ for the first trimester of pregnancy and supplements are available over the counter from pharmacies

It is important for researchers and public health programme evaluators to measure maternal FA supplement intake in order to assess compliance with supplementation guidelines and to assess the success of public health interventions aimed at increasing FA supplementation uptake amongst women planning a pregnancy. Folate biomarkers such as plasma folate (PF) (a short-term indicator) and erythrocyte folate (a longer term indicator) provide an objective measure of maternal folate intake ${ }^{(7,15,26-27)}$ yet are not routinely measured in epidemiological studies due to cost and subject burden ${ }^{(28)}$. Epidemiological studies commonly rely on maternal self-report as a measure of FA supplement intake, which may be unreliable and prone to participant error and recall bias ${ }^{(27,29)}$.

Little is known about the folate status of NZ pregnant women and its relation to FA and micronutrient supplement intake during pregnancy. The aim of the present study was to examine the association between reported supplemental FA intake and folate biomarkers (PF) in a sample of NZ pregnant women. Data from a cohort of NZ pregnant women at 15 weeks' gestation enrolled in the Screening for Pregnancy Endpoints (SCOPE) prospective study ${ }^{(30)}$ were used for this analysis. Specific objectives were to (1) describe reported maternal FA supplement use at 15 weeks' gestation, (2) analyse maternal PF levels at 15 weeks' gestation in order to confirm reported FA supplement use, (3) investigate which socio-demographic and lifestyle factors were associated with PF at 15 weeks' gestation and (4) examine any additional effect of reported multivitamin intake on PF levels at 15 weeks' gestation.

\section{Materials and methods \\ Screening for Pregnancy Endpoints study}

The present study is a retrospective cross-sectional analysis using data and blood samples from the NZ participants of the international SCOPE study conducted in healthy nulliparous women. The primary aim of SCOPE was to produce clinically useful screening tests to detect first-time mothers at high risk of pre-eclampsia, spontaneous preterm birth and/or having small-for-gestational age babies. SCOPE contains detailed data about FA supplementation, including dosage and gestation, collected concurrently with maternal blood samples at the participant research visit at 15 weeks' gestation. This provided the opportunity to assess maternal FA supplement intake and measure PF at the same time point (15 weeks' gestation). SCOPE data also included a wide range of socio-demographic and lifestyle factors ${ }^{(31)}$.

\section{Participant recruitment and selection}

Participants (healthy nulliparous Auckland women with singleton pregnancies) were recruited between 2004 and 2009: www.scopestudy.net. The SCOPE study was registered with the Australia New Zealand Clinical Trials Registry (ID $82254)^{(30)}$. Details of the SCOPE study methodology have previously been published ${ }^{(31)}$. In short, women were excluded if they had major risk factors for pre-eclampsia, spontaneous preterm birth and small-for-gestational age births ${ }^{(31)}$.

\section{Ethics}

Ethical approval was obtained from the NZ ethics committee AKX/02/00/364, and all participants provided written informed consent.

\section{Blood sample collection}

Non-fasting blood samples were collected into EDTA vacutainers at 15 weeks' gestation by trained research midwives and centrifuged at $3000 \mathrm{rpm}(2400 \mathrm{~g})$ for $10 \mathrm{~min}$ at $4{ }^{\circ} \mathrm{C}$. Plasma was aliquoted into lavender-capped barcoded cryotubes and scanned into the SCOPE database. Samples were then placed immediately into temperature-monitored freezers at The University of Auckland at $-80^{\circ} \mathrm{C}$ for storage.

\section{Plasma folate analysis}

At the time of folate analysis, samples were shipped on dry ice to the Human Nutrition Laboratories, University of Otago, New Zealand. PF was analysed using the microbiological assay in a 96-well plate and using chloramphenicol-resistant Lactobacillus rhamnosus (ATCC 7469), according to the methods of O'Broin \& Kelleher $^{(32)}$ and Molloy \& Scott ${ }^{(33)}$ (Costar 3596, Corning Inc.). Calibration curves were produced with eleven concentration points from 0 to $0 \cdot 1 \mathrm{pmol} /$ well using 5-methyltetrahydrofolate ((6S)-5-methyl-5,6,7,8-tetrahydropterolyl-L-glutamic acid, sodium salt; Merck Eprova). Plasma was diluted 1 in 75 by adding $40 \mu \mathrm{l}$ of plasma to $2.96 \mathrm{ml}$ of $0.5 \%$ sodium ascorbate solution. Turbidity was measured by absorbance at $590 \mathrm{~nm}$ on a Varioskan LUX multimode microplate reader (Thermo Scientific), and a cubic spline (Quartic Excel Macro, Interactive Design Services Pty) was applied to the calibration curve. Quality control (QC) criteria were as follows: a high, medium and low pooled serum (The National Institute of Standards and Technology Standard Reference Material 1955) were included on each plate and are expressed as mean: high $49 \cdot 2$ (sD 3.4) nmol/l; medium 28.5 (sD 1.8) nmol/l and low $14 \cdot 8(\mathrm{sD} 0 \cdot 9) \mathrm{nmol} / \mathrm{l}$. If all the QC results were within mean (SD 2) limits, the assay was accepted; if more than one of the QC results were outside the mean (SD 2 ) limits or any of the QC results were outside the mean (SD 3) limits, then the assay was rejected. Results from assay runs that passed QC were recorded only if the $\mathrm{CV}$ of all four replicates (two at each dilution) were below $15 \%$. If the CV of the four replicates was above $15 \%$, the largest outlier could be deleted and the result recorded as long as the CV of the three remaining replicates was below $10 \%$, otherwise the sample measurement was repeated.

\section{Statistical analysis}

We used $\chi^{2}$ tests to examine the differences in FA supplement use (yes/no) and maternal socio-demographic and lifestyle factors (all categorical). PF results were non-normally distributed and positively skewed, and log transformed prior to analysis. Pearson's correlation coefficient was used to examine the univariable association between $\mathrm{PF}(\mathrm{nmol} / \mathrm{l})$ and self-reported 
average daily FA supplement dose $(\mu \mathrm{g} / \mathrm{d})$ (continuous variables). ANOVA was used to investigate the differences in mean PF levels between FA users and non-users. ANOVA was also used to investigate the difference between participants who reported their FA supplementation intake from FA supplements alone, $v$. FA from a multivitamin supplement (or from both). To investigate the differences in mean PF levels by FA dose, daily dose (total from FA supplements plus any FA contained in multivitamin supplements) was categorised as follows: 0, 1-399, 400799, 800, 801-1999 and $\geq 2000 \mu \mathrm{g} / \mathrm{d}$, and mean PF in each category compared using ANOVA. Univariable linear regression was used to investigate the association between maternal socio-demographic, lifestyle and dietary variables, reported daily FA supplementation dose and PF levels, and the $\beta$-coefficient with $95 \%$ CI presented. Predictor variables were selected $a$ priori and included FA supplementation daily dose (total dose from FA supplements plus any FA contained in multivitamins), maternal age category, ethnicity, BMI category (normal weight, overweight and obese), smoking status (no/yes), alcohol intake (no/yes), socio-economic index (SEI) category (SEI scores were divided into three categories of equivalent score range, with a higher score representing a higher SEI) ${ }^{(34)}$, education level (>12 years $v$. $\leq 12$ years), high green leafy vegetable intake (no/yes for $\geq 3$ times/d), high fruit intake (no/yes for $\geq 3$ times/d), multivitamin use (no/yes) and vegetarianism (nonvegetarian, demi-vegetarian (may eat fish) and vegetarian). Gestation at measurement was also included as a predictor variable because PF can vary with gestational age ${ }^{(35)}$. Multiple linear regression was used to examine the independent association between FA supplementation daily dose and PF, adjusting for sample age (at the time of analysis) as well as for potential sociodemographic and dietary confounders that were associated with $\mathrm{PF}$ at a significance level of $P<0 \cdot 1$ in the univariable analysis.

The significance level was set at $5 \%$. All statistical analyses were conducted using STATA version 5 (StataCorp).

\section{Results}

Of the 2032 participants included in the SCOPE Auckland cohort, 1960 were eligible for inclusion in the present study, and the PF samples were available for a total of 1921 (98\%) of these participants. Participant characteristics are described in Table 1. The average maternal age was 30 years (sD 4 years 9 months). The majority of participants were NZ/other European, with the remaining participants identifying as Maori, Asian, Indian, Pacific and other non-European. Two-thirds of participants had more than 12 years' schooling and nearly two-thirds had a BMI $<25 \mathrm{~kg} / \mathrm{m}^{2}$.

\section{Folic acid supplementation intake}

Of the women in this cohort, $67 \%$ ( $n$ 1284) reported taking FA supplements in the pre-conception period, and $97 \%$ ( $n$ 1855) reported taking FA supplements in the first trimester. However, only $48 \%$ ( $n$ 916) of participants reported taking FA supplements as recommended by the NZ Ministry of Health (i.e. $800 \mu \mathrm{g}$ of FA daily for at least 4 weeks prior to conception and for the first 12 weeks of pregnancy) ${ }^{(25)}$. The majority of participants $(73 \% ; n$ 1408) reported taking an FA supplement at 15 weeks' gestation. Of these women, $21 \%$ reported consuming FA as a single nutrient tablet, while $79 \%$ reported consuming FA-containing multivitamin supplements, or from both an FA supplement and a multivitamin supplement. Nearly all participants ( $n$ 1906; $99 \%$ ) in the present study had data on multivitamin intake, of which 1125 (59\%) reported taking a multivitamin supplement at 15 weeks. Very few participants ( $n$ 27; $1.4 \%$ ) reported taking multivitamins with no FA. The mean of FA dose reported at 15 weeks' gestation was 564 (SE 13.3) $\mu \mathrm{g} / \mathrm{d}$. Fig. 1 presents daily FA supplement dose categories $(\mu \mathrm{g} / \mathrm{d})$ reported by participants at 15 weeks' gestation. Of the FA supplement users, $16 \%$ were taking less than $400 \mu \mathrm{g} / \mathrm{d}$. Of the total participants, $56 \%$ (and $76 \%$ of FA supplement users) reported taking at least $800 \mu \mathrm{g} / \mathrm{d}$ (Fig. 1). Table 1 shows FA supplement use at 15 weeks based on socio-demographic and lifestyle factors of participants. Maternal age, ethnicity, SEI and smoking status were significantly associated with FA supplement use/non-use at 15 weeks' gestation.

\section{Socio-demographic, dietary and supplementation data collection}

Participants were interviewed face-to-face and examined by a trained research midwife at 15 weeks' gestation. Details about participant age, SEI (based on the New Socio-economic Index 1996, with a higher score representing a higher socio-economic status) ${ }^{(34)}$, family history and specific information on FA and multivitamin supplement use (pre-conception (defined as the 4 weeks before conception), in the first trimester and at 15 weeks' gestation) were collected via an interviewer-administered dietary and lifestyle questionnaire. Height and weight were measured at this visit and used to calculate BMI. BMI was then categorised as normal weight $\left(<25 \mathrm{~kg} / \mathrm{m}^{2}\right.$; underweight participants were included in this category for analysis purposes), overweight $\left(\geq 25\right.$ to $\left.<30 \mathrm{~kg} / \mathrm{m}^{2}\right)$ and obese $\left(\geq 30 \mathrm{~kg} / \mathrm{m}^{2}\right)$. Information on FA use (yes/no), multivitamin supplement use (yes/no) and type and brand used at 15 weeks was collected by the research midwife and entered at the time of the interview into an Internetaccessed, password-protected centralised database with a complete audit trail (MedSciNet $\mathrm{AB}$ ). To calculate the total daily dose of FA $(\mu \mathrm{g})$ taken by participants, researchers tabulated the amount of FA from FA supplements and FA-containing multivitamins. If participants reported taking less supplements daily, researchers added together total FA dose taken per week and divided it by 7 to calculate the daily dose. Participants were asked to bring their FA and vitamin supplements while visiting SCOPE to verify the reported supplementation information.

\section{Plasma folate levels}

Mean PF in the study population was 48.5 (sE 1.6) nmol/l.

Table 2 shows the mean PF by FA supplement usage (yes/no) at 15 weeks' gestation. Mean PF was significantly higher in women reporting FA supplementation (54.6 (se 1.5$) \mathrm{nmol} / \mathrm{l}$ ) compared with women reporting no FA supplementation $(35.1$ ( $\mathrm{se} 1.6) \mathrm{nmol} / \mathrm{l} ; P<0 \cdot 001$ ). There was no significant difference in mean $\mathrm{PF}$ between participants who took FA alone, compared with participants who took an 
Table 1. Association between maternal folic acid supplement use and socio-demographic/lifestyle factors at 15 weeks' gestation (Numbers and percentages)

\begin{tabular}{|c|c|c|c|c|c|c|c|}
\hline \multirow[b]{3}{*}{ Characteristics } & & & \multicolumn{4}{|c|}{ Folic acid supplement use } & \multirow[b]{3}{*}{$P^{\star}$} \\
\hline & \multicolumn{2}{|c|}{ Total } & \multicolumn{2}{|c|}{ No } & \multicolumn{2}{|c|}{ Yes } & \\
\hline & $n$ & $\%$ & $n$ & $\%$ & $n$ & $\%$ & \\
\hline All participants & 1921 & 100 & 513 & $26 \cdot 7$ & 1408 & $73 \cdot 3$ & - \\
\hline Maternal age (years) & & & & & & & $<0.001$ \\
\hline$<25$ & 199 & 10 & 88 & 44 & 111 & 56 & \\
\hline 25 to $<30$ & 554 & $2 \cdot 9$ & 139 & 25 & 415 & 75 & \\
\hline 30 to $<35$ & 836 & 43.5 & 203 & 24 & 633 & 76 & \\
\hline$\geq 35$ & 332 & $17 \cdot 3$ & 83 & 25 & 249 & 75 & \\
\hline Ethnicity & & & & & & & $<0.001$ \\
\hline NZ/other European & 1625 & $84 \cdot 6$ & 417 & 26 & 1208 & 74 & \\
\hline Asian & 96 & $5 \cdot 0$ & 18 & 19 & 78 & 81 & \\
\hline Other non-European & 31 & 1.6 & 12 & 39 & 19 & 61 & \\
\hline Maori & 60 & $3 \cdot 1$ & 25 & 32 & 35 & 68 & \\
\hline Indian & 76 & 4.0 & 23 & 30 & 53 & 70 & \\
\hline Pacific & 40 & 1.7 & 18 & 45 & 15 & 55 & \\
\hline Education level (years) & & & & & & & 0.791 \\
\hline$>12$ & 1249 & 65 & 336 & 27 & 913 & 73 & \\
\hline$\leq 12$ & 672 & 35 & 177 & 26 & 495 & 74 & \\
\hline SEI† & & & & & & & 0.029 \\
\hline $18-41$ & 422 & 22 & 134 & 32 & 288 & 68 & \\
\hline $42-65$ & 1344 & 70 & 339 & 25 & 1005 & 75 & \\
\hline $66-90$ & 155 & 8 & 40 & 26 & 115 & 74 & \\
\hline BMI category $\left(\mathrm{kg} / \mathrm{m}^{2}\right)$ & & & & & & & 0.241 \\
\hline$<25$ & 1175 & $61 \cdot 2$ & 307 & 26 & 868 & 74 & \\
\hline $25-<30$ & 537 & 28.0 & 140 & 26 & 397 & 74 & \\
\hline$\geq 30$ & 209 & $10 \cdot 9$ & 66 & 32 & 143 & 68 & \\
\hline Smoking status & & & & & & & 0.008 \\
\hline No & 1849 & $96 \cdot 3$ & 484 & 26 & 1365 & 74 & \\
\hline Yes & 72 & 3.7 & 29 & 40 & 43 & 60 & \\
\hline Alcohol use & & & & & & & 0.764 \\
\hline No & 1821 & 94.8 & 485 & 27 & 1336 & 73 & \\
\hline Yes & 100 & $5 \cdot 2$ & 28 & 28 & 72 & 72 & \\
\hline High fruit intake & & & & & & & 0.912 \\
\hline No & 876 & $45 \cdot 6$ & 235 & 27 & 641 & 73 & \\
\hline Yes & 1045 & 54.4 & 278 & 27 & 767 & 73 & \\
\hline High green leafy vegetable intake & & & & & & & 0.059 \\
\hline No & 1685 & $87 \cdot 7$ & 462 & 27 & 1223 & 73 & \\
\hline Yes & 236 & $12 \cdot 3$ & 51 & 22 & 185 & 78 & \\
\hline Vegetarianism & & & & & & & 0.333 \\
\hline Non-vegetarian & 1815 & $96 \cdot 4$ & 491 & 27 & 1324 & 73 & \\
\hline Demi-vegetarian $\ddagger$ & 67 & 3.6 & 13 & 19 & 54 & 81 & \\
\hline Vegetarian & 39 & 2 & 9 & 23 & 30 & 77 & \\
\hline
\end{tabular}

SEI, socio-economic index

* $P$ value for trend: $\chi^{2}$ test

† SEl score was divided into three categories of equal range for this comparison. A higher score represents a higher SEI.

$\ddagger$ Demi-vegetarians may eat fish.

FA-containing multivitamin or an FA supplement plus a multivitamin $(P=0.918)$ (Table 3$)$.

PF was significantly positively correlated with reported FA dose: $r 0.41(P<0 \cdot 05)$. A sensitivity analysis was conducted to examine the influence of the high-dose FA $(\geq 2000 \mu \mathrm{g} / \mathrm{d})$ supplement users; removal of these participants did not significantly affect the correlation. Mean PF levels by daily FA dose categories are shown in Table 4. PF increased significantly across the overall dose categories $(P<0 \cdot 001)$; however, no differences in mean PF were observed between dose categories 400-799, 800 and 801-1999 $\mu \mathrm{g} / \mathrm{d}$.

Univariable regression showed that the reported FA daily dose category (total dose from both FA supplements and from FA contained in multivitamin supplements) was significantly associated with PF (Table 4). Multiple linear regression showed that after adjusting for measured confounders, the significant association between FA supplementation dose and PF remained and was relatively unchanged (Table 4). Univariable regression showed that PF at 15 weeks' gestation was significantly associated with maternal age, gestation at measurement, ethnicity, BMI category, cigarette smoking, SEI, high intake of green leafy vegetables, multivitamin use and vegetarian status at 15 weeks' gestation (Table 4). Of the confounders in the multivariable regression model, only maternal age, ethnicity, vegetarian status and BMI category remained significant. Mean PF increased with advancing maternal age category. Mean PF was highest amongst Asian participants, followed by NZ/other European participants; Pacific participants had the lowest mean PF level (60\% lower 


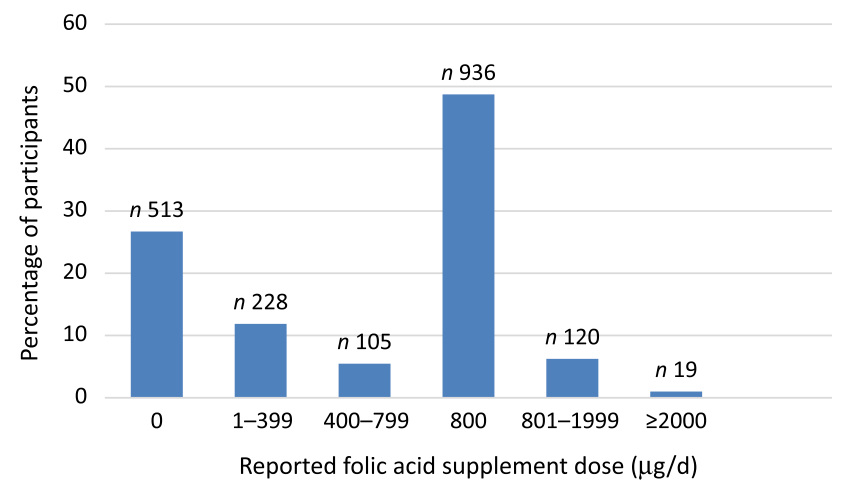

Fig. 1. Reported maternal folic acid supplement dose $(\mu \mathrm{g} / \mathrm{d})$ at 15 weeks' gestation.

than NZ/other European participants). Obese participants had a significantly lower mean PF than normal weight participants, and vegetarians had a significantly higher mean PF than non-vegetarians (Table 4).

\section{Discussion}

\section{Summary of findings}

In the present study of 1921 pregnant NZ women, almost three quarters of participants were taking an FA supplement in the early second trimester, with more than three quarters of these taking a daily dose of $\geq 800 \mu \mathrm{g}$ (the amount recommended in NZ up to the end of the first trimester) ${ }^{(25)}$. Despite the high rate of FA supplement use in both the first trimester and the early second trimester, only half of the participants in our study were meeting the Ministry of Health FA supplementation recommendation of $800 \mu \mathrm{g} / \mathrm{d}$ pre-conception and during the first trimester ${ }^{(25)}$. Three-quarters of FA supplement users at 15 weeks' gestation took FA as part of or alongside a multivitamin supplement. FA supplementation was significantly associated with PF levels at 15 weeks' gestation, with those reporting FA supplement use having a significantly higher (56\%) mean PF concentration compared with those reporting no use. Maternal report of FA supplement intake was confirmed by PF. FA daily dose, maternal age, gestation at measurement, ethnicity, BMI and vegetarian status were significantly associated with $\mathrm{PF}$.

\section{Interpretation}

Findings from the present study confirm that maternal report of FA supplementation was a reliable proxy measure of FA supplement use in this sample. The present study contributes to the limited data on the patterns of FA supplement use and its associated PF concentrations among NZ pregnant women. To the authors' knowledge, this is the first study to describe maternal FA supplementation intake and PF concentrations in the early second trimester in a large cohort of NZ pregnant women.

The high proportion (73\%) of women who reported taking FA supplementation in the early second trimester was somewhat surprising. In NZ, guidelines recommend that women take FA supplements $(800 \mu \mathrm{g} / \mathrm{d})$ in the pre-conception period and up
Table 2. Plasma folate by folic acid supplement use at 15 weeks' gestation (Numbers and percentages; mean values with their standard errors)

\begin{tabular}{|c|c|c|c|c|c|}
\hline \multirow{2}{*}{$\begin{array}{l}\text { Folic acid } \\
\text { supplement use }\end{array}$} & \multicolumn{2}{|c|}{ Participants } & \multicolumn{2}{|c|}{$\begin{array}{l}\text { Plasma folate } \\
\qquad(\mathrm{nmol} / \mathrm{l})\end{array}$} & \multirow[b]{2}{*}{$P$} \\
\hline & $n$ & $\%$ & Mean & SE & \\
\hline All participants & 1921 & 100 & 48.5 & 1.6 & - \\
\hline No & 513 & $26 \cdot 7$ & $35 \cdot 1$ & 1.6 & Ref \\
\hline Yes & 1408 & $73 \cdot 3$ & $54 \cdot 6$ & 1.5 & $<0.001$ \\
\hline
\end{tabular}

Ref, reference category.

Table 3. Plasma folate by type of folic acid supplement at 15 weeks' gestation

(Numbers and percentages; mean values with their standard errors)

\begin{tabular}{|c|c|c|c|c|c|}
\hline \multirow{2}{*}{$\begin{array}{l}\text { Type of folic acid supplement } \\
\text { used }(n 1394)^{*}\end{array}$} & \multirow[b]{2}{*}{$n$} & \multirow[b]{2}{*}{$\%$} & \multicolumn{2}{|c|}{$\begin{array}{l}\text { Plasma folate } \\
\qquad(\mathrm{nmol} / \mathrm{l})\end{array}$} & \multirow[b]{2}{*}{$P$} \\
\hline & & & Mean & $\mathrm{SE}$ & \\
\hline Folic acid supplement alone & 293 & 21 & $55 \cdot 2$ & $1 \cdot 7$ & Ref \\
\hline $\begin{array}{l}\text { Folic acid taken as part of or } \\
\text { alongside a multivitamin }\end{array}$ & 1101 & 79 & $54 \cdot 6$ & 1.5 & 0.918 \\
\hline
\end{tabular}

Ref, reference category.

* Seventeen values missing for multivitamin status and removal of $n 27$ : multivitamin with no folic acid.

to 12 weeks' gestation ${ }^{(25)}$, yet a substantial number of women in the present study were taking FA supplements beyond this time point. Although the percentage of participants in the present study who reported taking FA supplements in the second trimester (73\%) is somewhat higher than that reported in another recently studied cohort of NZ pregnant women $(56.7 \%)^{(36)}$, the findings of both show that a significant number of $\mathrm{NZ}$ pregnant women report taking FA supplementation beyond the first trimester. The NZ Ministry of Health acknowledges that supplementing with $400 \mu \mathrm{g}$ daily is sufficient to reduce the risk of NTD. However, the only FA tablets that are currently available as registered medicines (i.e., subsidised) and, therefore, available over the counter from pharmacies in NZ contain either 800 or $5000 \mu \mathrm{g}$ FA. As such, women are recommended to take the FA tablets that are registered as medicines, to reduce the occurrence or re-occurrence of NTD. Nonetheless, results indicated that more than three quarters (79\%) of supplement users were taking FA in the form of or alongside multivitamins. A possible explanation for the extended use of FA supplementation observed in our study is that the majority of women were taking FA as part of a multivitamin, which they purchased themselves, and continued to use beyond the first trimester. In comparison, the smaller proportion of participants who took an FA-only supplement tablet (which is subsidised in NZ from pre-conception until the end of the first trimester) would probably have discontinued its use after this recommended period.

Overall, 39\% of the total participants in the present study were not meeting the WHO Antenatal Care Guidelines' recommendation of $400 \mu \mathrm{g}$ supplemental FA daily throughout pregnancy $^{(24)}$. Moreover, given the lack of mandatory FA fortification of the food supply in NZ, pregnant women probably 
Table 4. Association between plasma folate and socio-demographic, lifestyle and supplementation variables at 15 weeks' gestation (Numbers and percentages; mean values with their standard errors; $\beta$-coefficients and $95 \%$ confidence intervals)

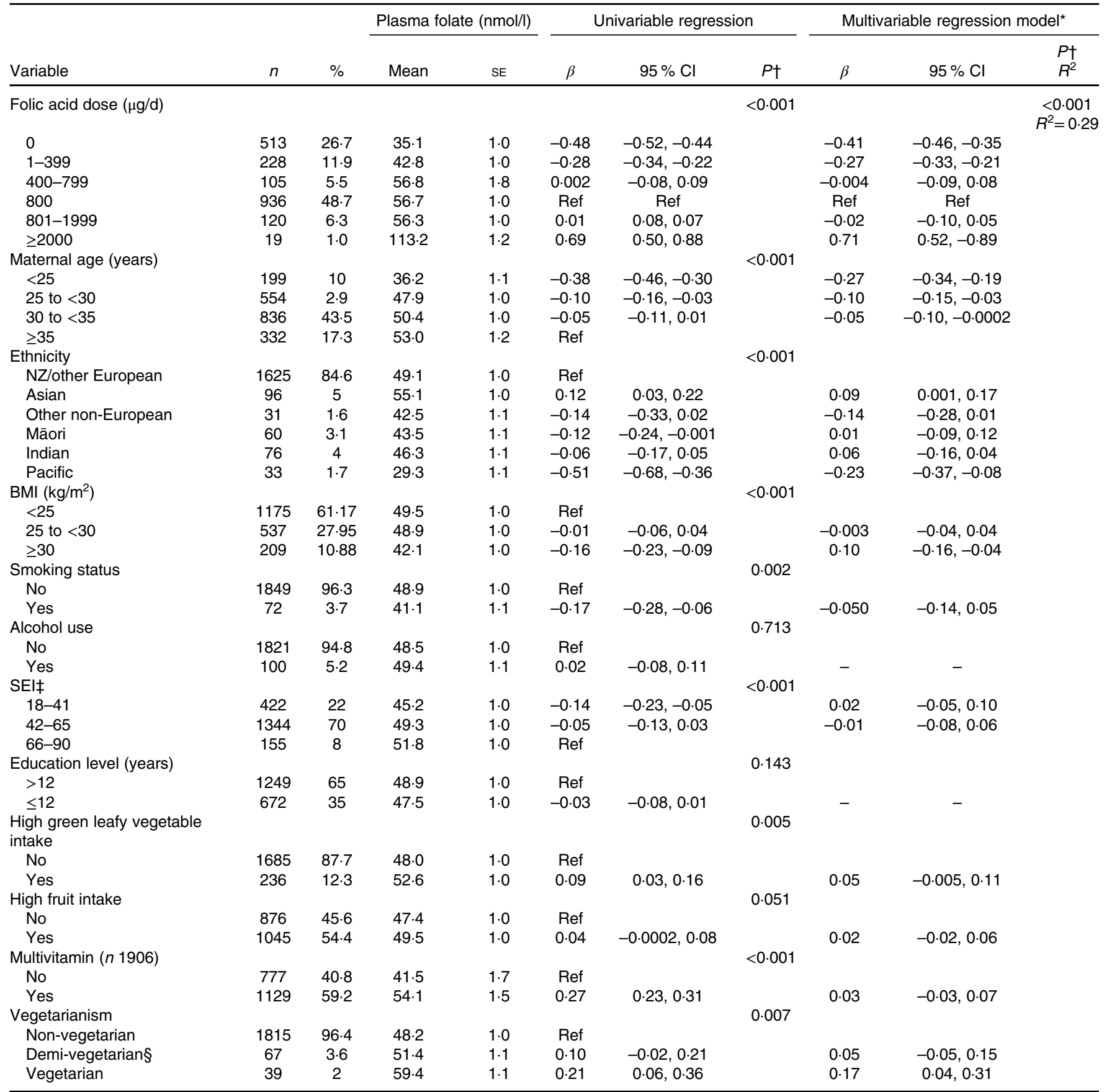

$n$, number of participants; Ref, reference category; SEI, socio-economic index.

* Independent variable: plasma folate. Dependent variable: folic acid supplement dose category. Model also adjusted for gestational age at measurement.

† $P$ rob $>F$ (for the multivariable regression model the $P$ value (Prob $>F$ ) for the overall model is shown).

$\ddagger$ SEI score was divided into three categories of equal range for this comparison. A higher score represents a higher SEl.

$\S$ Demi-vegetarians may eat fish.

do not meet the Recommended Dietary Intake of $600 \mu \mathrm{g} / \mathrm{d}^{(37)}$. Mean dietary intake of folate among NZ pregnant women $(212 \mu \mathrm{g} / \mathrm{d})$ published nearly 20 years ago indicated that intake was well below the recommended levels ${ }^{(38)}$. Studies from other countries with a similar policy of supplementation and voluntary
FA fortification have shown that FA intake from voluntarily fortified foods has been found to contribute at least $100 \mu \mathrm{g} / \mathrm{d}^{(39)}$. Therefore, further contemporary longitudinal research is needed to determine the total dietary folate and FA intake by NZ pregnant women and women of childbearing age. This should 
include FA intake from both supplements and voluntary fortified food, in order to gain a better understanding of whether supplementation should be recommended beyond the first trimester of pregnancy.

PF levels were higher in FA supplement users $v$. non-users, consistent with the previous findings from other studies examining folate biomarkers in pregnant women ${ }^{(40-48)}$. Similarly, a nationally representative study of $\mathrm{NZ}$ adults found that FAcontaining supplement users had a $48 \%$ higher mean serum folate concentration compared with non-users ${ }^{(49)}$. The correlation between reported FA supplementation dose and PF seen in the present study is also similar to that found in a Dutch study conducted in the early second trimester of pregnancy $(r 0.49$; $P=0 \cdot 001)^{(50)}$.

In the present study at the time of reporting of supplement intake and blood sampling, a significant association between FA dose category and PF was observed. However, given the cross-sectional and observational study design, the absolute dose effect is unknown. These results support the findings of other similar studies ${ }^{(50-52)}$, and randomised controlled trails ${ }^{(53-55)}$. Further research into the kinetics of FA on PF concentrations in pregnancy and its associated outcomes is needed.

Similar to other studies, maternal age, gestational age and ethnicity were associated with PF concentrations in pregnancy ${ }^{(40,45,56)}$. In contrast, reported multivitamin use was not significantly associated with PF in the multivariable analysis, suggesting no additional benefits conferred from other micronutrients present in the supplement on short-term folate status. Of particular importance, the findings that mean PF differed significantly between ethnic groups (with a significantly lower PF level observed in Pacific participants) warrants further exploration. A higher BMI category was also significantly negatively associated with PF in the present study, in contrast with the findings by Liu $e t$ $a l .{ }^{(45)}$ and Ma et $a l .{ }^{(57)}$. This difference is probably due to the use of only two categories of BMI in their analyses (normal BMI $v$. overweight), whereas the present study also included a third (BMI $>30 \mathrm{~kg} / \mathrm{m}^{2}$ (obese)) category. Similar to the present study, Cheng et al. ${ }^{(40)}$ also included a third (obese) category and found a significantly lower mean PF level in the obese group compared with the normal BMI group. Previous studies examining folate levels in women of childbearing age have found lower PF but higher erythrocyte folate levels in the obese women, suggesting that obesity might influence folate metabolism and that ideally both should be measured pre-conception so that personalised advice can be provided ${ }^{(58)}$.

While the WHO provides a reference range for optimal erythrocyte folate levels (for the prevention of NTD), no optimal PF reference range exists for pregnancy. Therefore, we are unable to comment on the overall sufficiency of folate status in our sample. Future studies examining the folate status of NZ pregnant women and women of childbearing age should investigate erythrocyte folate to provide information on longer term folate status and how levels compare with the WHO recommendation for optimal erythrocyte folate $(\geq 906 \mathrm{nmol} / \mathrm{l})$ for women of childbearing age ${ }^{(59)}$. Likewise, the influence of FA-containing multivitamins $v$. FA supplements alone on erythrocyte folate status, homocysteine concentrations and pregnancy outcomes in
NZ women should be investigated to understand whether micronutrients other than FA provide additional benefits.

\section{Strengths and limitations}

SCOPE study provided an opportunity to investigate the association between a range of socio-demographic and lifestyle variables, FA supplementation and PF concentrations in a large sample size of over 1900 pregnant women. Socio-demographic, lifestyle variables and blood samples were collected at 15 weeks' gestation by trained research midwives, according to the standard operating procedures. FA supplementation was verified directly, including brand names and doses. In addition, multivitamin use was also documented, allowing the capture of all supplemental FA uptake as well as providing an opportunity to investigate the effect of multivitamins on the association between FA supplementation and PF levels.

The present study has a number of limitations. These data are not reflective of the general pregnant population of NZ. Our analysis was conducted on an opportunistic population of participants from one geographical region (Auckland, NZ). The cohort was predominantly of NZ/other European ethnicity, which comprises less than $60 \%$ of the NZ population. This limits the generalisability of the findings to other $\mathrm{NZ}$ areas and cultural groups. The study collected a range of supplement information but did not measure dietary folate intake. However, the consumption of green leafy vegetable and high fruit intake was included in the analysis of the effect of FA supplementation on PF. The assessment of maternal folate status in the present study was based on a single measurement of $\mathrm{PF}^{(58)}$. Erythrocyte folate, a longer term indicator of folate status ${ }^{(24,58)}$, was not analysed, and warrants investigation in future studies. However, the aim of the present study was to examine the reported intake of FA supplementation concurrently with blood sample analysis, and PF is the most applicable measure of the recent $\mathrm{FA}$ intake ${ }^{(60)}$. In the present study, blood was obtained in a non-fasting state to increase study participation. However, the non-fasting blood collection could have falsely elevated PF concentrations. Nonetheless, given the relatively small difference between the non-fasting and fasting PF state, non-fasting PF is an acceptable measure for population-based estimates ${ }^{(61)}$. One further limitation of our study is the categorisation of normal weight, overweight and obesity based on maternal BMI measured at 15 weeks of gestation, because an objective measure of pre-pregnancy BMI was not available. The Institute of Medicine Guidelines ${ }^{(62)}$ recommend a weight gain between 0.5 and $2 \mathrm{~kg}$ in the first trimester, and as such, this change in weight may have affected the accuracy of classification.

\section{Conclusion}

The present study of NZ women in early second trimester of pregnancy found a significant association between reported FA supplementation intake and maternal PF levels. This positive relationship demonstrates that self-reported FA supplemental intake in this sample population is indeed a reliable proxy indicator of FA supplement use. 
The significant difference observed in mean PF across ethnic groups (in particular the lower PF levels seen in Pacific participants) and across BMI categories (lower PF seen in obese women) suggests that further research should focus on assessing the differences in long-term folate status between these groups, to inform targeted interventions. Studies examining how these levels might be associated with pregnancy outcomes will provide valuable insight for targeted public health campaigns around FA supplementation in pregnancy and will help ensure best pregnancy outcomes. Such research will also help inform whether FA supplementation should be recommended in NZ beyond the first trimester, to be in line with the WHO Antenatal Care Guidelines ${ }^{(24)}$.

\section{Acknowledgements}

The authors would like to thank the SCOPE study participants and research midwives. The authors would like to thank Rennae Taylor, SCOPE Study Coordinator, for advice and assistance with accessing samples for analysis. The authors would also like to thank Karl Bailey and Michelle Harper at the University of Otago for conducting the sample analysis.

The study was funded by the University of Auckland Faculty of Medical and Health Sciences Faculty Research Development Fund (grant/award no. 370927).

R. B., L. M., J. T., C. W. and L. H. planned the study. R. B. analysed the data, LH advised on the PF analysis interpretation, and J. T. checked the statistical analysis. R. B. wrote the first draft of the manuscript. L. G., J. T., C. W. and L. H. contributed to the interpretation and critically reviewed the manuscript. All authors contributed to and approved the final version of the manuscript.

There are no conflicts of interest.

\section{References}

1. Mahan LK, Escott-Stump S, Raymond JL, et al. (2012) Krause's Food \& the Nutrition Care Process, 13th ed. St. Louis, MI: Mosby, Elsevier Health Sciences.

2. Smith AD, Kim YI \& Refsum H (2008) Is folic acid good for everyone? Am J Clin Nutr 87, 517-533.

3. Talaulikar VS \& Arulkumaran S (2011) Folic acid in obstetric practice: a review. Obstet Gynecol Surv 66, 240-247.

4. Kalhan SC (2013) One-carbon metabolism, fetal growth and long-term consequences. Nestle Nutr Inst Workshop Ser $\mathbf{7 4}$, $127-138$.

5. Lassi ZS, Salam RA, Haider BA, et al. (2013) Folic acid supplementation during pregnancy for maternal health and pregnancy outcomes. Cochrane Database Syst Rev, issue 3, CD006896.

6. Nelms M, Sucher K \& Long S (2007) Nutrition Therapy and Pathophysiology, 1st ed. Belmont, CA: The Thomson Corporation.

7. Word Health Organization (2015) Guideline: optimal serum and red blood cell folate concentrations in women of reproductive age for prevention of neural tube defects. Geneva: World Health Organisation. www.who.int/nutrition/publications/ guidelines/optimalserum_rbc_womenrep_tubedefects/en/ (accessed May 2018).

8. Williams PJ, Bulmer JN, Innes BA, et al. (2011) Possible roles for folic acid in the regulation of trophoblast invasion and placental development in normal early human pregnancy. Biol Reprod 84, 1148-1153.
9. Kalhan SC \& Marczewski SE (2012) Methionine, homocysteine, one carbon metabolism and fetal growth. Rev Endocr Metab Disord 13, 109-119.

10. Bulloch RE, Lovell AL, Jordan VMB, et al. (2018) Maternal folic acid supplementation for the prevention of preeclampsia: a systematic review and meta-analysis. Paediatr Perinat Epidemiol 32, 346-357.

11. Mantovani E, Filippini F, Bortolus R, et al. (2014) Folic acid supplementation and preterm birth: results from observational studies. Biomed Res Int 2014, 481914.

12. Catov JM, Bodnar LM, Olsen J, et al. (2011) Periconceptional multivitamin use and risk of preterm or small-for-gestationalage births in the Danish national birth cohort. Am J Clin Nutr 94, 906-912.

13. Bodnar LM, Himes KP, Venkataramanan R, et al. (2010) Maternal serum folate species in early pregnancy and risk of preterm birth. Am J Clin Nutr 92 , 864-871.

14. Vahratian A, Siega-Riz AM, Savitz DA, et al. (2004) Multivitamin use and the risk of preterm birth. Am J Epidemiol 160, 886-892.

15. van Uitert EM \& Steegers-Theunissen RP (2013) Influence of maternal folate status on human fetal growth parameters. Mol Nutr Food Res 57, 582-595.

16. Ramakrishnan U, Grant F, Goldenberg T, et al. (2012) Effect of women's nutrition before and during early pregnancy on maternal and infant outcomes: a systematic review. Paediatr Perinat Epidemiol 26, Suppl. 1, 285-301.

17. Scholl TO \& Johnson WG (2000) Folic acid: influence on the outcome of pregnancy. Am J Clin Nutr 71, 5 Suppl., 1295S-303S

18. Wen SW, Chen X, Rodger M, et al. (2008) Folic acid supplementation in early second trimester and the risk of preeclampsia. Obstet Gynecol 198, 45.e1-45.e7.

19. Carberry AE, Gordon A, Bond DM, et al. (2014) Customised versus population-based growth charts as a screening tool for detecting small for gestational age infants in low-risk pregnant women. Cochrane Database Syst Rev, issue 5, CD008549.

20. Hodgetts V, Morris R, Francis A, et al. (2015) Effectiveness of folic acid supplementation in pregnancy on reducing the risk of small-for-gestational age neonates: a population study, systematic review and meta-analysis. BJOG 122, 478-90.

21. Payne B, Magee LA \& von Dadelszen P (2011) Assessment, surveillance and prognosis in pre-eclampsia. Best Pract Res Clin Obstet Gynaecol 25, 449-462.

22. Abalos E, Cuesta C, Grosso AL, et al. (2013) Global and regional estimates of preeclampsia and eclampsia: a systematic review. Eur J Obstet Gynecol Reprod Biol 170, 1-7.

23. Khan KS, Wojdyla D, Say L, et al. (2006) WHO analysis of causes of maternal death: a systematic review. Lancet 367, 1066-1074.

24. World Health Organization (2016) WHO recommendations on antenatal care for a positive pregnancy experience. Geneva: World Health Organization. www.who.int/reproductivehealth/ publications/maternal_perinatal_health/anc-positive-pregnancyexperience/en/ (accessed May 2018).

25. Ministry of Health (2008) Food and Nutrition Guidelines for Healthy Pregnant and Breastfeeding Women: A Background Paper. Wellington: Ministry of Health. www.health.govt.nz/ publication/food-and-nutrition-guidelines-healthy-pregnantand-breastfeeding-women-background-paper (accessed May 2018).

26. Bailey LB \& Hausman DB (2018) Folate status in women of reproductive age as basis of neural tube defect risk assessment. Ann N Y Acad Sci 1414, 82-95.

27. Bailey LB, Stover PJ, McNulty H, et al. (2015) Biomarkers of nutrition for development: folate review. J Nutr 145, 1636S-1680S. 
28. Hedrick VE, Dietrich AM, Estabrooks PA, et al. (2012) Dietary biomarkers: advances, limitations and future directions. Nutrition J 11, 109.

29. Subar AF, Freedman LS, Tooze JA, et al. (2015) Addressing current criticism regarding the value of self-report dietary data, 2. J Nutr, 145, 2639-2645.

30. Australian New Zealand Clinical Trials Registry (ANZCTR) (2007) Screening for Pregnancy Endpoints: Preeclampsia, Growth Restricted Baby and Spontaneous Preterm Birth. ACTRN 12607000551493. http://www.anzctr.org.au/trial_ view.aspx?ID=82254 (accessed May 2018).

31. North RA, McCowan LM, Dekker GA, et al. (2011) Clinical risk prediction for pre-eclampsia in nulliparous women: development of model in international prospective cohort. BMJ 342, d1875.

32. O'Broin S \& Kelleher B (1992) Microbiological assay on microtitre plates of folate in serum and red cells. J Clin Pathol 45, 344-7

33. Molloy AM \& Scott JM (1997) Microbiological assay for serum, plasma, and red cell folate using cryopreserved, microtiter plate method. Methods Enzymol 281, 43-53.

34. Galbraith C, Jenkin G, Davis P, et al. (1996) New Zealand Socioeconomic Index 1996: User's Guide. Wellington, NZ: Statistics New Zealand.

35. Cikot R, Steegers-Theunissen R, Thomas C, et al. (2001) Longitudinal vitamin and homocysteine levels in normal pregnancy. BrJ Nutr 85, 49-58.

36. Teixeira JA, Castro TG, Wall CR, et al. (2018) Determinants of folic acid supplement use outside national recommendations for pregnant women: results from the Growing Up in New Zealand cohort study. Public Health Nutr 21, 2183-2192.

37. National Health and Medical Research Council (NHMRC), Australian Government Department of Health and Ageing, New Zealand Ministry of Health (2006) Nutrient Reference Values for Australia and New Zealand. Canberra: National Health and Medical Research Council; Wellington: Ministry of Health.

38. Russell DG, Parnell WR, Wilson NC, et al. (1999) NZ Food: NZ People. Key Results of the 1997 National Nutrition Survey. Wellington: Ministry of Health.

39. Pentieva K, Selhub J, Paul L, et al. (2016) Evidence from a randomized trial that exposure to supplemental folic acid at recommended levels during pregnancy does not lead to increased unmetabolized folic acid concentrations in maternal or cord blood. J Nutr 146, 494-500.

40. Cheng TL, Mistry KB, Wang G, et al. (2018) Folate nutrition status in mothers of the Boston birth cohort, sample of a US urban low-income population. Am J Public Health 108, 799-807.

41. Brantsæter AL, Haugen M, Hagve TA, et al. (2007) Self-reported dietary supplement use is confirmed by biological markers in the Norwegian Mother and Child Cohort Study (MoBa). Ann Nutr Metab 51, 146-154.

42. Colapinto CK, O'Connor DL, Dubois L, et al. (2012) Folic acid supplement use is the most significant predictor of folate concentrations inCanadian women of childbearing age. Appl Physiol Nutr Metab 37, 284-292.

43. Matsuzaki M, Haruna M, Ota E, et al. (2008) Dietary folate intake, use of folate supplements, lifestyle factors, and serum folate levels among pregnant women in Tokyo, Japan. $J$ Obstet Gynaecol Res 34, 971-979.

44. Burton A, Wilson S \& Gillies AJ (2001) Folic acid: is self-reported use of supplements accurate? J Epidemiol Community Health 55, 841-842.

45. Liu J, Gao L \& Zhang Y (2015) Plasma folate levels in early to mid pregnancy after a nation-wide folic acid supplementation program in areas with high and low prevalence of neural tube defects in China. Teratology 103, 501-508.

46. Murto T, Yngve A, Skoog Svanberg A, et al. (2017) Compliance to the recommended use of folic acid supplements for women in Sweden is higher among those under treatment for infertility than among fertile controls and is also related to socioeconomic status and lifestyle. Food Nutr Res 61, 1334483.

47. Roth C, Bjørke-Monsen AL, Reichborn-Kjennerud T, et al. (2013) Use of folic acid supplements in early pregnancy in relation to maternal plasma levels in week 18 of pregnancy. Mol Nutr Food Res 57, 653-660.

48. Pfeiffer CM, Sternberg MR, Fazili Z, et al. (2015) Folate status and concentrations of serum folate forms in the US population: National Health and Nutrition Examination Survey 2011-2. Br J Nutr 113, 1965-1977.

49. Bradbury KE, Williams SM, Mann JI, et al. (2013) Estimation of serum and erythrocyte folate concentrations in the New Zealand adult population within a background of voluntary folic acid fortification. J Nutr 144, 68-74.

50. Sikkens JJ, van Eijsden M, Bonsel GJ, et al. (2011) Validation of self-reported folic acid use in a multiethnic population: results of the Amsterdam Born Children and their Development study. Public Health Nutr 14, 2022-2028.

51. Yang Q, Cogswell ME, Hamner HC, et al. (2009) Folic acid source, usual intake, and folate and vitamin B-12 status in US adults: National Health and Nutrition Examination Survey (NHANES) 2003-2006. Am J Clin Nutr 91, 64-72.

52. Yeung L, Yang Q \& Berry RJ (2008) Contributions of total daily intake of folic acid to serum folate concentrations. JAMA $\mathbf{3 0 0}$, 2486-2487.

53. Duffy ME, Hoey L \& Hughes CF (2013) Biomarker responses to folic acid intervention in healthy adults: a meta-analysis of randomized controlled trials. Am J Clin Nutr 99, 96-106.

54. Caudill MA, Cruz AC, Gregory III JF, et al. (1997) Folate status response to controlled folate intake in pregnant women. J Nutr 127, 2363-2370.

55. Hursthouse NA, Gray AR, Miller JC, et al. (2011) Folate status of reproductive age women and neural tube defect risk: the effect of long-term folic acid supplementation at doses of $140 \mu \mathrm{g}$ and $400 \mu \mathrm{g}$ per day. Nutrients 3, 49-62.

56. Larroque B, Kaminski M, Lelong N, et al. (1992) Folate status during pregnancy: relationship with alcohol consumption, other maternal risk factors and pregnancy outcome. Eur $J$ Obstet Gynecol 43, 19-27.

57. Ma R, Wang L, Jin L, et al. (2017) Plasma folate levels and associated factors in women planning to become pregnant in a population with high prevalence of neural tube defects. Birth Defects Res 109, 1039-1047.

58. Maffoni S, De Giuseppe R, Stanford FC, et al. (2017) Folate status in women of childbearing age with obesity: a review. Nutr Res Rev 30, 265-71.

59. World Health Organization (2015) Serum and Red Blood Cell Folate Concentrations for Assessing Folate Status in Populations. Vitamin and Mineral Nutrition Information System. Geneva: World Health Organization. https://www. who.int/nutrition/publications/micronutrients/indicators_ serum_RBC_folate/en/ (accessed June 2018).

60. Bailey RL, Fulgoni VL, Taylor CL, et al. (2017) Correspondence of folate dietary intake and biomarker data. Am JClin Nutr $\mathbf{1 0 5}$, 1336-1343

61. Pfeiffer CM, Zhang M \& Jabbar S (2018) Framework for laboratory harmonization of folate measurements in low-and middleincome countries and regions. Ann N Y Acad Sci 1414, 96-108.

62. Institute of Medicine (2009) Weight Gain During Pregnancy: Re-examining the Guidelines. Washington, DC: The National Academies Press. 